\title{
AN IDENTITY FOR THE SCHUR COMPLEMENT OF A MATRIX
}

DOUGLAS E. CRABTREE AND EMILIE V. HAYNSWORTH ${ }^{1}$

1. Introduction. Let $A=\left(a_{i j}\right)$ be an $n \times n$ complex matrix, and suppose that $B$ is a nonsingular principal submatrix of $A$. We define the Schur complement of $B$ in $A$, denoted by $(A / B)$, as follows: Let $\hat{A}$ be the matrix obtained from $A$ by a simultaneous permutation of rows and columns which puts $B$ into the upper left corner of $\hat{A}$.

$$
A=\left[\begin{array}{ll}
B & E \\
D & G
\end{array}\right] .
$$

Then $(A / B)=G-D B^{-1} E$.

Since

$$
\begin{aligned}
\operatorname{det} A & =\operatorname{det} \hat{A}=\operatorname{det}\left[\begin{array}{cc}
I & 0 \\
-D B^{-1} & I
\end{array}\right] \operatorname{det} \hat{A}=\operatorname{det}\left[\begin{array}{cc}
I & 0 \\
-D B^{-1} & I
\end{array}\right]\left[\begin{array}{ll}
B & E \\
D & G
\end{array}\right] \\
& =\operatorname{det}\left[\begin{array}{cc}
B & E \\
0 & G-D B^{-1} E
\end{array}\right]=\operatorname{det} B \operatorname{det}\left(G-D B^{-1} E\right),
\end{aligned}
$$

we see that

$$
\operatorname{det} A=\operatorname{det} B \operatorname{det}(A / B) \text {. }
$$

This result is known as Schur's formula.

In case $A$ is Hermitian, Haynsworth [5] has shown that the inertia of $A$ can be determined from the inertia of any nonsingular principal submatrix of $A$ together with that of its Schur complement. Other applications and properties of the Schur complement will appear in a later paper.

In $\$ 2$ of this note, we prove that the Schur complement can also be constructed using quotients of minors of $A$. Details on this method of construction and its relation to partitioned matrices and $M$ matrices can be found in [1], [2], [3].

In $\S 3$, this construction is used to prove a quotient identity for the Schur complement: $(A / B)=((A / C) /(B / C))$.

2. Elements of the Schur complement. The notation $A\left(i_{1}, \cdots, i_{p}\right.$; $j_{1}, \cdots, j_{p}$ ) denotes the submatrix of $A$ formed using rows $i_{1}, \cdots, i_{p}$

Received by the editors October 2, 1968.

${ }^{1}$ The work of the second author was done under Contract DA-91-591-EUC-3686 of the U.S. Army with the Institute of Mathematics, University of Basel. The author wishes to thank Professor A. M. Ostrowski for very helpful discussions. 
and columns $j_{1}, \cdots, j_{p}$. For principal submatrices we abbreviate this notation to $A\left(i_{1}, \cdots, i_{p}\right)$.

Lemma. Let $C=A(1, \cdots, k)$ be a nonsingular leading principal submatrix of $A$. Let $F=\left(f_{i j}\right)$ be the matrix with elements

$$
\begin{aligned}
f_{i j}=\operatorname{det} A(1, \cdots, k, i ; 1, \cdots, k, j) / \operatorname{det} C \\
\\
\quad(i, j=k+1, \cdots, n) .
\end{aligned}
$$

Then $F=(A / C)$, the Schur complement of $C$ in $A$.

PROOF. Let $b_{i j}$ denote the bordered minor

$$
b_{i j}=\operatorname{det} A(1, \cdots, k, i ; 1, \cdots, k, j)=f_{i j} \operatorname{det} C .
$$

With $A$ partioned in the form

$$
A=\left[\begin{array}{ll}
C & E \\
D & G
\end{array}\right],
$$

let $D^{(i)}$ denote the $(i-k)$ th row of $D$, and let $E_{(j)}$ denote the $(j-k)$ th column of $E$. Thus

$$
D^{(i)}=\left[a_{i 1}, \cdots, a_{i k}\right] \quad(i=k+1, \cdots, n)
$$

and

$$
E_{(j)}=\left[\begin{array}{c}
a_{1 j} \\
\vdots \\
a_{k j}
\end{array}\right] \quad(j=k+1, \cdots, n) .
$$

Then for $i, j=k+1, \cdots, n$,

$$
b_{i j}=\operatorname{det}\left[\begin{array}{ll}
C & E_{(j)} \\
D^{(i)} & a_{i j}
\end{array}\right] .
$$

By Schur's formula, $b_{i j}=\left(a_{i j}-D^{(i)} C^{-1} E_{(j)}\right)(\operatorname{det} C)$. Thus

$$
f_{i j}=\left(a_{i j}-D^{(i)} C^{-1} E_{(j)}\right) .
$$

But these are precisely the elements of the matrix $(A / C)=G-D C^{-1} E$, so the lemma is proved.

We remark that the lemma allows us to restate a result contained in [1, Lemma 1]: The Schur complement of an $M$-matrix is an $M$ matrix.

\section{The quotient property of the Schur complement.}

Theorem. If $B$ is a nonsingular principal submatrix of $A$, and $C$ 
is a nonsingular principal submatrix of $B$, then $(B / C)$ is a nonsingular principal submatrix of $(A / C)$, and $(A / B)=((A / C) /(B / C))$.

Proof. We assume without loss of generality that $C=A(1, \cdots, k)$ and $B=A(1, \cdots, p)$, with $k<p$. Let $V=(A / C)$, of order $n-k$, and let $W=(B / C)$, of order $p-k$. (We label the rows and columns of $V$ from $k+1$ to $n$. Similarly, the indices for $W$ are $i, j=k+1, \cdots, p$, while for $(V / W)$ we use $i, j=p+1, \cdots, n$.) It follows from the lemma that $W$ is a principal submatrix of $V$. Moreover, $W$ is nonsingular, since by Schur's formula,

$$
\operatorname{det} W=(\operatorname{det} B) /(\operatorname{det} C) \text {. }
$$

Now let $\bar{V}=(\operatorname{det} C) V$. For $i, j=p+1, \cdots, n$ we have

$$
\begin{aligned}
(V / W)_{i, j} & =\operatorname{det} V(k+1, \cdots, p, i ; k+1, \cdots, p, j) / \operatorname{det} W \\
& =\operatorname{det} C \operatorname{det} V(k+1, \cdots, p, i ; k+1, \cdots, p, j) / \operatorname{det} B \\
& =\operatorname{det} \bar{V}(k+1, \cdots, p, i ; k+1, \cdots, p, j) / \operatorname{det} B(\operatorname{det} C)^{p-k} .
\end{aligned}
$$

Since the elements of the matrix $\bar{V}$ are bordered minors from $A$, Sylvester's determinant identity [4] enables us to express the determinant of any square submatrix of $\bar{V}$ in terms of the corresponding submatrix of $A$. In particular,

$$
\begin{aligned}
\operatorname{det} \bar{V}(k+1, \cdots, p & , i ; k+1, \cdots, p, j) \\
& =(\operatorname{det} C)^{p-k} \operatorname{det} A(1, \cdots, p, i ; 1, \cdots, p, j) .
\end{aligned}
$$

Thus

$$
(V / W)_{i, j}=\operatorname{det} A(1, \cdots, p, i ; 1, \cdots, p, j) / \operatorname{det} B,
$$

which, by the lemma, equals $(A / B)_{i, j}$.

\section{REFERENCES}

1. Douglas E. Crabtree, Applications of $M$-matrices to nonnegative matrices, Duke Math. J. 33 (1966), 197-208.

2. - Characteristic roots of M-matrices, Proc. Amer. Math. Soc. 17 (1966), 1435-1439.

3. —, A matrix identity, Amer. Math. Monthly 75 (1968), 648-649. 33.

4. F. R. Gantmacher, The theory of matrices, Vol. 1, Chelsea, New York, 1959, p.

5. Emilie V. Haynsworth, Determination of the inertia of a partitioned Hermitian matrix, Linear Algebra and Appl. 1 (1968), 73-81.

Amherst College and

AUbuRn University 